\title{
Zeta functions of finite groups by enumerating subgroups
}

\author{
Yumiko Hironaka \\ Department of Mathematics, \\ Faculty of Education and Integrated Sciences, Waseda University \\ Nishi-Waseda, Tokyo, 169-8050, JAPAN
}

abstract

For a finite group $G$, we consider the zeta function $\zeta_{G}(s)=\sum_{H}|H|^{-s}$, where $H$ runs over the subgroups of $G$. First we give simple examples of abelian $p$-group $G$ and non-abelian $p$-group $G^{\prime}$ of order $p^{m}, m \geq 3$ for odd $p$ (resp. $\left.2^{m}, m \geq 4\right)$ for which $\zeta_{G}(s)=\zeta_{G^{\prime}}(s)$. Hence we see there are many non-abelian groups whose zeta functions have symmetry and Euler product, like the case of abelian groups. On the other hand, we show that $\zeta_{G}(s)$ determines the isomorphism class of $G$ within abelian groups, by estimating the number of subgroups of abelian $p$-groups. Finally we study the problem which abelian $p$-group is associated with a non-abelian group having the same zeta function.

\section{$\S 0$}

Let us consider zeta functions associated to finite groups by enumerating their subgroups. For a group $G$, one may consider the following zeta functions if they convergent in some region

$$
\zeta_{G}(s)=\sum_{n=1}^{\infty} \frac{a_{n}(G)}{n^{s}}, \quad \zeta_{G}^{*}(s)=\sum_{n=1}^{\infty} \frac{a_{n}^{*}(G)}{n^{s}},
$$

where $s \in \mathbb{C}$ and $a_{n}(G)$ (resp. $a_{n}^{*}(G)$ ) is the number of subgroups of order $n$ (resp. index $n$ ) in $G$. If $G=\mathbb{Z}, \zeta_{G}^{*}(s)$ is nothing but the Riemann zeta function, and this is an example of Solomon's zeta functions for lattices. It seems to be natural to consider $\zeta_{G}^{*}(s)$ in general and there are many studies in that direction. As for finitely generated (infinite) groups, there are interesting

Mathematical Subject Classification 2010: 20E07(primary), 11M41, $20 \mathrm{~K} 01$.

This research is partially supported by Grant-in-Aid for Scientific Research (C): 24540031. 
results by F. J. Grunewald, M. P. F. du Sautoy, et al. (e.g. [GSS], [Sau], SG] .

In this paper we concentrate on finite groups and study $\zeta_{G}(s)$. There is no essential difference between the two zeta functions, since there is a simple relation

$$
\zeta_{G}(s)=\frac{1}{|G|^{s}} \zeta_{G}^{*}(-s), \quad(\text { for a finite group } G) .
$$

Moreover, the duality for finite abelian groups yields that $\zeta_{G}(s)=\zeta_{G}^{*}(s)$, hence one has

$$
\zeta_{G}(s)=\frac{1}{|G|^{s}} \zeta_{G}(-s), \quad(\text { for a finite abelian group } G)
$$

In the following we consider only finite groups. It is natural to ask

[A] whether $\zeta_{G}(s)$ determines the isomorphism class of $G$ or not,

[B] whether the symmetry (0.3) is essential for abelian groups or not.

In $\S 1$, we will construct examples of abelian groups and non-abelian groups that have the same zeta functions, which shows Questions [A] and [B] are negative in general. On the other hand, we will show Question [A] is affirmative within abelian groups in $\S 2$. For abelian groups, Question [A] is reduced to enumerating subgroups of abelian $p$-groups and combinatorial studies, and there is a close relation to automorphic forms and local densities of matrices (cf. Remark 2.8). Finally in $\S 3$, we give a partial answer for the problem which abelian $p$-group is associated with a non-abelian group having the same zeta function.

\section{$\S 1$}

The zeta function $\zeta_{G}(s)$ is a polynomial with non-negative integral coefficients of $p^{-s}$ with various $p$, where and henceforth we denote by $p$ a prime number. We start with an easy observation.

Proposition 1.1 (1) The zeta function $\zeta_{G}(s)$ determines the order $|G|$ of $G$.

(2) If $|G|$ and $\left|G^{\prime}\right|$ are coprime, then $\zeta_{G \times G^{\prime}}(s)=\zeta_{G}(s) \times \zeta_{G^{\prime}}(s)$.

(3) The fact only 1 appears as coefficients of $\zeta_{G}(s)$ is equivalent to that $G$ is cyclic. 
Proof. The statements (1) and (3) are clear. Under the assumption of (2), any subgroup of $G \times G^{\prime}$ is written uniquely as a direct product of the form $H \times H^{\prime}$ for some subgroups $H$ of $G$ and $H^{\prime}$ of $G^{\prime}$.

We say $\zeta_{G}(s)$ has an Euler product if it can be decomposed as

$$
\zeta_{G}(s)=\prod_{p \| G \mid} f_{p}\left(p^{-s}\right), \quad f_{p}(X) \in \mathbb{C}[X] .
$$

Then we have

Proposition $1.2 \zeta_{G}(s)$ has an Euler product if and only if $G$ is a nilpotent group, i.e. $G$ is a direct product of its Sylow p-subgroups. The Euler product (1.1) is uniquely determined under the condition $f_{p}(0)=1$ for any $p$ dividing $|G|$.

Proof. Assume $\zeta_{G}(s)$ has an Euler product of the shape (1.1). Since $\prod_{p} f_{p}(0)=1\left(=a_{1}(G)\right)$, we may assume the constant term of $f_{p}(X)$ is equal to 1 for any prime divisor $p$ of $|G|$. For such a $p$, the coefficient of $X^{m}$ in $f_{p}(X)$ must coincide with $a_{p^{m}}(G)$, hence it is a non-negative integer. Denote by $c_{p}$ the leading coefficient of $f_{p}(X)$. Then $\prod_{p|| G \mid} c_{p}=1$ yields $c_{p}=1$ for any $p$, which means there is only one normal Sylow $p$-subgrop in $G$ for each $p$ and $G$ is nilpotent. The converse and the uniqueness of the Euler product are clear.

By the above proof, we see that the decomposition (1.1) is unique if one takes the constant terms of the Euler factors as 1 and actually given by polynomials with non-negative integral coefficients.

Here we give an example which does not have an Euler product. Take an odd integer $n(\geq 3)$ and consider the dihedral group of order $2 n$

$$
D_{n}=\left\langle\sigma, \tau \mid \sigma^{n}=\tau^{2}=1, \tau \sigma \tau=\sigma^{-1}\right\rangle .
$$

Then

$$
\begin{aligned}
\zeta_{D_{n}}(s) & =1+n \cdot 2^{-s}+\sum_{d \mid n, d \neq 1}\left(d^{-s}+(2 d)^{-s}\right) \\
& =(n-1) \cdot 2^{-s}+\left(1+2^{-s}\right) \prod_{p \mid n} f_{p}\left(p^{-s}\right)
\end{aligned}
$$

where

$$
f_{p}(X)=X^{m_{p}}+X^{m_{p}-1}+\cdots+X+1, \quad n=\prod_{p \mid n} p^{m_{p}}
$$

We construct some $p$-groups which are counter examples for Problems [A] and $[\mathrm{B}]$. We denote by $C_{n}$ the cyclic group of order $n$. For a prime $p$ and 
positive integers $m$ and $n$ such that $m>n$, we define a non-abelian group of order $p^{m+n}$ as follows:

$$
G_{p}(m, n)=\left\langle\sigma, \tau \mid \sigma^{p^{m}}=\tau^{p^{n}}=1, \tau \sigma \tau^{-1}=\sigma^{1+p^{m-1}}\right\rangle
$$

Proposition 1.3 Assume $G_{p}(m, n)$ is given as above and $m \geq 3$ if $p=2$.

(1) The non-abelian group $G_{p}(m, n)$ and the abelian group $C_{p^{m}} \times C_{p^{n}}$ have the same zeta function, and it satisfies the symmetry (0.3).

(2) The p-groups of order less than or equal to $p^{2}$ if $p$ is odd (resp. $2^{3}$ if $p=2$ ) are determined by their zeta functions.

Proof. (1) Let us write $G=G_{p}(m, n)$ and assume $m \geq 3$ if $p=2$.

Since we see

$$
\left(\sigma^{i} \tau^{j}\right)^{r}=\sigma^{i\left(r+\frac{r(r-1)}{2} j p^{m-1}\right)} \tau^{r j}, \quad i, j, r \in \mathbb{N},
$$

we have

$$
\left(\sigma^{i} \tau^{j}\right)^{p}= \begin{cases}\sigma^{p i} \tau^{p j} & \text { if } p \neq 2 \\ \sigma^{2 i\left(1+j 2^{m-2}\right)} \tau^{2 j} & \text { if } p=2\end{cases}
$$

Thus we see

$$
\operatorname{ord}\left(\sigma^{i} \tau^{j}\right)=p^{m} \text { if and only if } p \nmid i \text {. }
$$

If $p$ Xij, the group $\left\langle\sigma^{i} \tau^{p^{s} j}\right\rangle$ contains an element $\sigma^{i^{\prime}} \tau^{p^{s}}$ for some $i^{\prime}$ not divided by $p$. Further, for $i, \ell$ not divided by $p$, we see that $\left\langle\sigma^{i} \tau^{p^{s}}\right\rangle=\left\langle\sigma^{\ell} \tau^{p^{s}}\right\rangle$ if and only if $i \equiv \ell\left(\bmod p^{n-s}\right)$. Hence all the subgroups of $G$ isomorphic to $C_{p^{m}}$ are given as follows:

$$
\left\langle\sigma^{i} \tau^{p^{s}}\right\rangle, \quad 0 \leq s \leq n, 1 \leq i \leq p^{n-s}, p \nmid i .
$$

For $i$ not divided by $p$, setting $\widetilde{\sigma}=\sigma^{i} \tau^{p^{s}}$, we have $G=\langle\widetilde{\sigma}, \tau\rangle$ with the same relations for $\sigma$ and $\tau$. There is a natural bijective correspondence between the set of subgroups of $G$ containing $\langle\widetilde{\sigma}\rangle$ and that of $G /\langle\widetilde{\sigma}\rangle \cong C_{p^{n}}$, and there is only one subgroup $K_{r}$ of $G$ such that $\left(K_{r}:\langle\widetilde{\sigma}\rangle\right)=p^{r}$ for each $r$ with $0 \leq r \leq n$.

On the other hand, any subgroup of $G$ without elements of order $p^{m}$ is contained in $\left\langle\sigma^{p}, \tau\right\rangle \cong C_{p^{m-1}} \times C_{p^{n}}$.

Hence we see that the subgroups of $G$ naturally and bijectively correspond to those of $C_{p^{m}} \times C_{p^{n}}$, hence their zeta functions coincide.

(2) It is well-known that any $p$-group of order less than or equal to $p^{2}$ is isomorphic to $C_{p}, C_{p^{2}}$ or $C_{p} \times C_{p}$, and any 2-group of order 8 is isomorphic to one of the following groups

$$
\begin{aligned}
& C_{8}, \quad C_{4} \times C_{2}, \quad C_{2} \times C_{2} \times C_{2}, \\
& D_{4}=\left\langle\sigma, \tau \mid \sigma^{4}=\tau^{2}=1, \tau \sigma \tau=\sigma^{-1}\right\rangle\left(=G_{2}(2,1)\right), \\
& Q_{2}=\left\langle\sigma, \tau \mid \sigma^{4}=1, \sigma^{2}=\tau^{2}, \tau \sigma \tau^{-1}=\sigma^{-1}\right\rangle .
\end{aligned}
$$


By a direct calculation, we have

$$
\begin{aligned}
& \zeta_{C_{p}}(s)=1+p^{-s}, \\
& \zeta_{C_{p^{2}}}(s)=1+p^{-s}+p^{-2 s}, \\
& \zeta_{C_{p} \times C_{p}}(s)=1+(p+1) p^{-s}+p^{-2 s}, \\
& \zeta_{C_{8}}(s)=1+2^{-s}+2^{-2 s}+2^{-3 s}, \\
& \zeta_{C_{4} \times C_{2}}=1+3 \cdot 2^{-s}+3 \cdot 2^{-2 s}+2^{-3 s}, \\
& \zeta_{C_{2} \times C_{2} \times C_{2}}(s)=1+7 \cdot 2^{-s}+7 \cdot 2^{-2 s}+2^{-3 s}, \\
& \zeta_{D_{4}}(s)=1+5 \cdot 2^{-s}+3 \cdot 2^{-2 s}+2^{-3 s}, \\
& \zeta_{Q_{2}}(s)=1+2^{-s}+3 \cdot 2^{-2 s}+2^{-3 s} .
\end{aligned}
$$

Remark 1.4 As for the number $a_{p^{k}}\left(G_{p}(m, n)\right)=a_{p^{k}}\left(C_{p^{m}} \times C_{p^{n}}\right)$, one can easily obtain directly, or by using Lemma 2.3 in $\S 2$ for $\lambda=(m, n) \in \Lambda_{2}^{+}$and $N_{k}(\lambda)=a_{p^{k}}\left(C_{p^{m}} \times C_{p^{n}}\right)$.

For a positive integer $n$ and a prime $p$, we denote by $v_{p}(n)$ the maximal exponent of $p$ dividing $n$. By Propositions 1.2 and 1.3, we obtain the next theorem, which gives a negative answer for Problems [A] and [B]. We note here that the groups $D_{4}$ and $Q_{2}$ are not abelian and their zeta functions are not symmetric (cf. (1.5)) ).

Theorem 1.5 (1) For a positive integer $n$ such that $v_{p}(n) \geq 3$ for some odd prime $p$ or $v_{2}(n) \geq 4$, there exist abelian groups and non-abelian nilpotent groups of order $n$ which have the same zeta functions.

(2) For a positive integer $n$ such that $v_{p}(n) \leq 2$ for each odd prime $p$ and $v_{2}(n) \leq 3$, any nilpotent group $G$ of order $n$ is determined up to isomorphism by its zeta function $\zeta_{G}(s)$, and it satisfies the symmetry (0.3) if and only if $G$ is abelian.

\section{$\S 2$}

In this section we enumerate the number of subgroups for abelian $p$-groups. It is well-known that every finite abelian $p$-group is isomorphic to one of $G_{\lambda}$ of the following type:

$$
G_{\lambda}=\mathbb{Z} / p^{\lambda_{1}} \mathbb{Z} \oplus \cdots \oplus \mathbb{Z} / p^{\lambda_{n}} \mathbb{Z}, \quad \lambda \in \Lambda_{n}^{+}
$$


where

$$
\Lambda^{+}=\bigsqcup_{n \geq 1} \Lambda_{n}^{+}, \quad \Lambda_{n}^{+}=\left\{\lambda \in \mathbb{Z}^{n} \mid \lambda_{1} \geq \lambda_{2} \geq \cdots \geq \lambda_{n}>0\right\}
$$

Hereafter we write $\zeta_{\lambda}(s)$ instead of $\zeta_{G_{\lambda}}(s)$. We denote by $N_{k}(\lambda)$ the number of subgroups of $G_{\lambda}$ of order $p^{k}$. We note $N_{k}(\lambda)=0$ if $k<0$ or $k>|\lambda|$, for any $\lambda \in \Lambda^{+}$.

The following is clear for $\lambda \in \Lambda_{n}^{+}$:

$$
\begin{aligned}
& \left|G_{\lambda}\right|=p^{|\lambda|}, \quad|\lambda|=\sum_{i=1}^{n} \lambda_{i}, \\
& \zeta_{\lambda}(s)=\sum_{k=0}^{|\lambda|} N_{k}(\lambda) p^{-k s}, \\
& N_{1}(\lambda)=\left(p^{n}-1\right) /(p-1)=p^{n-1}+p^{n-2}+\cdots+p+1, \\
& N_{k}(\lambda)=N_{|\lambda|-k}(\lambda), 0 \leq k \leq|\lambda|,
\end{aligned}
$$

where (2.6) is a restatement of the duality of abelian groups (cf. (0.3)). Moreover, it is known that $N_{k}(\lambda)$ is a polynomial in $p$ with integral coefficients and unimodal, i.e., $N_{k}(\lambda)-N_{k-1}(\lambda)$ is a polynomial in $p$ with nonnegative coefficients for $1 \leq k \leq|\lambda| / 2$ (cf. [Bu1], Remark 2.7).

We prepare some notations. Let $\lambda \in \Lambda_{n}^{+}$. Define

$$
\begin{aligned}
& c_{\ell}(\lambda)=\lambda_{\ell+1}+\lambda_{\ell+3}+\cdots+\left(\begin{array}{lll}
\lambda_{n} & \text { if } n \neq \ell & (\bmod 2) \\
\lambda_{n-1} & \text { if } n \equiv \ell & (\bmod 2)
\end{array}\right), 1 \leq \ell \leq n-1, \\
& c_{n}(\lambda)=0, \quad c_{-\ell}(\lambda)=|\lambda|-c_{\ell}(\lambda),
\end{aligned}
$$

in particular

$$
e v_{\lambda}=c_{1}(\lambda)=\sum_{i=1}^{[n / 2]} \lambda_{2 i}, \quad o d_{\lambda}=c_{-1}(\lambda)=\sum_{i=0}^{[n-1 / 2]} \lambda_{2 i+1},
$$

and we divide the interval $[0,|\lambda|]$ into $(2 n-1)$ small intervals $J_{\ell}(\lambda)$ as follows.

$$
\begin{aligned}
& J_{0}(\lambda)=\left[e v_{\lambda}, o d_{\lambda}\right], \\
& J_{\ell}(\lambda)=\left[c_{\ell+1}(\lambda), c_{\ell}(\lambda)\right], \quad J_{-\ell}(\lambda)=\left[c_{-\ell}(\lambda), c_{-(\ell+1)}(\lambda)\right], \quad(1 \leq \ell \leq n-1) .
\end{aligned}
$$

We also define

$$
a_{\ell}(\lambda)=\sum_{i=\ell+2}^{n}\left[\frac{i-\ell}{2}\right] \lambda_{i},(0 \leq \ell \leq n-1),
$$

where we understand $a_{n-1}(\lambda)=0$. 
For $\lambda \in \Lambda_{n}^{+},(n \geq 2)$, we define

$$
\lambda^{(\ell)}=\left(\lambda_{\ell+1}, \ldots, \lambda_{n-1}, \lambda_{n}\right) \in \Lambda_{n-\ell}^{+}, \quad 1 \leq \ell \leq n-1,
$$

and we set $\lambda^{\prime}=\lambda^{(1)}$ for simplicity. It is easy to see

$$
J_{\ell}(\lambda)=J_{0}\left(\lambda^{(\ell)}\right), \quad \ell \geq 1 \text {. }
$$

For a polynomial $g(t)$ in $t$, we denote by hterm $(g(t)), \operatorname{hdeg}(g(t))$ and htermcoeff $(g(t))$ its highest term, highest degree and highest coefficient in $t$, in order. We recall $N_{k}(\lambda)$ can be regarded as a polynomial of $p$, and set $H_{n}(\lambda)=\max \left\{\operatorname{hdeg}\left(N_{k}(\lambda)\right)|0 \leq k \leq| \lambda \mid\right\}$.

Theorem 2.1 The highest term of $N_{k}(\lambda), \lambda \in \Lambda_{n}^{+}$, as a polynomial in $p$ is given as

$$
\begin{aligned}
\operatorname{hterm}\left(N_{k}(\lambda)\right) & =C(n, \lambda ; k) p^{\ell k+a_{\ell}(\lambda)}, \quad \text { if } k \in J_{\ell}(\lambda), 0 \leq \ell \leq n-1, \\
\operatorname{hterm}\left(N_{k}(\lambda)\right) & =\operatorname{hterm}\left(N_{|\lambda|-k}(\lambda)\right), \quad \text { if }|\lambda| / 2 \leq k \leq|\lambda| \\
& \left(=C(n, \lambda ;|\lambda|-k) p^{-\ell k+\ell|\lambda|+a_{\ell}(\lambda)}, \quad \text { if } k \in J_{-\ell}(\lambda), \ell \geq 1\right) .
\end{aligned}
$$

Here $C(n, \lambda ; k)=1$ if $n \leq 2$, and in general

$$
\begin{aligned}
& C(n, \lambda ; k) \\
& = \begin{cases}C\left(n-\ell, \lambda^{(\ell)} ; k\right) & \text { if } k \in J_{\ell}(\lambda)=J_{0}\left(\lambda^{(\ell)}\right), \ell \geq 1, \\
\sum_{i=\max \left\{e v_{\lambda}, k-\lambda_{1}+\lambda_{2}\right\}}^{\min \left\{k, o d_{\lambda}-\lambda_{1}+\lambda_{2}\right\}} C\left(n-1, \lambda^{\prime} ; i\right) & \text { if } k \in J_{0}(\lambda) .\end{cases}
\end{aligned}
$$

Further, for each $\ell \geq 0, C(n, \lambda ; k), k \in J_{\ell}(\lambda)$ is unimodal and symmetric with respect to $\left|\lambda^{(\ell)}\right| / 2$ and $C\left(n, \lambda ; c_{\ell}(\lambda)\right)=1$. In particular, $H_{n}(\lambda)=a_{0}(\lambda)=$ $\sum_{i=2}^{n}\left[\frac{i}{2}\right] \lambda_{i}$.

Remark 2.2 We see the leading coefficients of smaller sizes appear repeatedly like a fractale, since $C(n, \lambda ; k)=C\left(n-\ell, \lambda^{(\ell)} ; k\right)$ for $k \in J_{\ell}(\lambda)$ with $\ell \geq 1$. The new coefficients appear for $k \in J_{0}(\lambda)$.

It may happen $J_{\ell}(\lambda)=\left\{c_{\ell}(\lambda)\right\}$, but $c_{\ell}(\lambda)>c_{\ell+2}(\lambda)$ for any $\ell,-n \leq \ell \leq n-2$. 
We give some examples of $C(n, \lambda ; k)$ for $k \in J_{0}(\lambda)$ :

$$
\begin{aligned}
& C(3, \lambda ; k)= \begin{cases}k-\lambda_{2}+1 & \text { if } k \in\left[\lambda_{2}, \lambda_{2}+\lambda_{3}\right] \\
\lambda_{3}+1 & \text { if } k \in\left[\lambda_{2}+\lambda_{3}, \lambda_{1}\right] \\
-k+\lambda_{1}+\lambda_{3}+1 & \text { if } k \in\left[\lambda_{1}, \lambda_{1}+\lambda_{3}\right],\end{cases} \\
& \text { or } \begin{cases}k-\lambda_{2}+1 & \text { if } k \in\left[\lambda_{2}, \lambda_{1}\right] \\
\lambda_{1}-\lambda_{2}+1 & \text { if } k \in\left[\lambda_{1}, \lambda_{2}+\lambda_{3}\right] \\
-k+\lambda_{1}+\lambda_{3}+1 & \text { if } k \in\left[\lambda_{2}+\lambda_{3}, \lambda_{1}+\lambda_{3}\right] .\end{cases} \\
& C(4, \lambda ; k)= \begin{cases}k-\lambda_{2}-\lambda_{4}+1 & \text { if } k \in\left[\lambda_{2}+\lambda_{4}, \lambda_{2}+\lambda_{3}\right] \\
\lambda_{3}-\lambda_{4}+1 & \text { if } k \in\left[\lambda_{2}+\lambda_{3}, \lambda_{1}+\lambda_{4}\right] \\
-k+\lambda_{1}+\lambda_{3}+1 & \text { if } k \in\left[\lambda_{1}+\lambda_{4}, \lambda_{1}+\lambda_{3}\right],\end{cases} \\
& \text { or } \begin{array}{ll}
k-\lambda_{2}-\lambda_{4}+1 & \text { if } k \in\left[\lambda_{2}+\lambda_{4}, \lambda_{1}+\lambda_{4}\right] \\
\lambda_{1}-\lambda_{2}+1 & \text { if } k \in\left[\lambda_{1}+\lambda_{4}, \lambda_{2}+\lambda_{3}\right] \\
-k+\lambda_{1}+\lambda_{3}+1 & \text { if } k \in\left[\lambda_{2}+\lambda_{3}, \lambda_{1}+\lambda_{3}\right] .
\end{array}
\end{aligned}
$$

The following lemma follows from a result of Stehling $([\mathrm{St}])$ and plays a key role for the proof of Theorem 2.1.

Lemma 2.3 For any $\lambda \in \Lambda_{n}^{+}$, set $\lambda^{\prime}=\left(\lambda_{2}, \ldots, \lambda_{n}\right) \in \Lambda_{n-1}^{+}$. Then one has

$$
N_{k}(\lambda)=\sum_{i=0}^{k} p^{i} N_{i}\left(\lambda^{\prime}\right)-\sum_{i=|\lambda|+1-k}^{\left|\lambda^{\prime}\right|} p^{i} N_{i}\left(\lambda^{\prime}\right), \quad 0 \leq k \leq|\lambda|,
$$

where the second summation appears only when $k>\lambda_{1}$.

Proof. For $\mu \in \Lambda_{n}^{+}$satisfying $\mu_{1}=\cdots=\mu_{i}>\mu_{i+1}$ for some $i \geq 1$, St, Theorem 1] claims

$$
N_{k}(\mu)=N_{k}\left(\mu^{*}\right)+p^{|\mu|-k} N_{k-\mu_{i}}\left(\mu^{\vee}\right),
$$

where $\mu^{*} \in \Lambda_{n}^{+}$defined by $\mu_{i}^{*}=\mu_{i}-1$ and $\mu_{j}^{*}=\mu_{j}$ except $j=i$, and $\mu^{\vee}=\left(\mu_{1}, \ldots, \mu_{i-1}, \mu_{i+1}, \ldots, \mu_{n}\right) \in \Lambda_{n-1}^{+}$. For any $\lambda \in \Lambda_{n}^{+}$, set $\widetilde{\lambda}=\left(\lambda_{1}+\right.$ $\left.1, \lambda_{2}, \ldots, \lambda_{n}\right) \in \Lambda_{n}^{+}$and $\lambda^{\prime}=\left(\lambda_{2}, \ldots, \lambda_{n}\right) \in \Lambda_{n-1}^{+}$. Then, since $\tilde{\lambda}^{*}=\lambda$ and $\tilde{\lambda}^{\vee}=\lambda^{\prime}$, one has by (2.17)

$$
N_{j}(\widetilde{\lambda})=N_{j}(\lambda)+p^{|\lambda|+1-j} N_{j-\lambda_{1}-1}\left(\lambda^{\prime}\right) .
$$

By the duality (2.6), and again by (2.17),

$$
\begin{aligned}
N_{j}(\widetilde{\lambda}) & =N_{|\lambda|+1-j}(\widetilde{\lambda})=N_{|\lambda|+1-j}(\lambda)+p^{j} N_{\left|\lambda^{\prime}\right|-j}\left(\lambda^{\prime}\right) \\
& =N_{j-1}(\lambda)+p^{j} N_{j}\left(\lambda^{\prime}\right) .
\end{aligned}
$$


Summing up RHD's of (2.18) and (2.19) from $j=1$ to $k$, one has (the terms $N_{j}(k)$ is cancelled for $\left.1 \leq j \leq k-1\right)$

$$
N_{k}(\lambda)+\sum_{j=1}^{k} p^{|\lambda|+1-j} N_{j-\lambda_{1}-1}\left(\lambda^{\prime}\right)=N_{0}(\lambda)+\sum_{j=1}^{k} p^{j} N_{j}\left(\lambda^{\prime}\right)
$$

hence (setting $i=|\lambda|+1-j$ in the LHS of the above), we have

$$
\begin{aligned}
N_{k}(\lambda) & =\sum_{j=0}^{k} p^{j} N_{j}\left(\lambda^{\prime}\right)-\sum_{i=|\lambda|+1-k}^{|\lambda|} p^{i} N_{|\lambda|-\lambda_{1}-i}\left(\lambda^{\prime}\right) \\
& =\sum_{j=0}^{k} p^{j} N_{j}\left(\lambda^{\prime}\right)-\sum_{i=|\lambda|+1-k}^{\left|\lambda^{\prime}\right|} p^{i} N_{\left|\lambda^{\prime}\right|-i}\left(\lambda^{\prime}\right) \\
& =\sum_{j=0}^{k} p^{j} N_{j}\left(\lambda^{\prime}\right)-\sum_{i=|\lambda|+1-k}^{\left|\lambda^{\prime}\right|} p^{i} N_{i}\left(\lambda^{\prime}\right),
\end{aligned}
$$

where the second summation appears only when $|\lambda|+1-k \leq\left|\lambda^{\prime}\right|$, i.e. $k>\lambda_{1}$.

For $r \in \Lambda_{1}^{+}$, since $G_{r}$ is a cyclic group of order $p^{r}$, one has

$$
N_{k}(r)=1, \quad \text { for } k \in J_{0}(r)=[0, r]
$$

Then, for $\mu \in \Lambda_{2}^{+}$, one has by Lemma 2.3

$$
\operatorname{hterm}\left(N_{k}(\mu)\right)= \begin{cases}p^{k} & \text { for } k \in J_{1}(\mu)=\left[0, \mu_{2}\right] \\ p^{\mu_{2}} & \text { for } k \in J_{0}(\mu)=\left[e v_{\mu}, o d_{\mu}\right]=\left[\mu_{2}, \mu_{1}\right] \\ p^{|\mu|-k} & \text { for } k \in J_{-1}(\mu)=\left[\mu_{1},|\mu|\right]\end{cases}
$$

We note the following useful relations :

$$
\begin{aligned}
& c_{-1}\left(\lambda^{\prime}\right)=\left|\lambda^{\prime}\right|-c_{1}\left(\lambda^{\prime}\right)=e v_{\lambda}, \quad c_{\ell-1}\left(\lambda^{\prime}\right)=c_{\ell}(\lambda),(\ell \geq 1) \\
& a_{\ell-1}\left(\lambda^{\prime}\right)=a_{\ell}(\lambda), \quad(\ell \geq 1) \\
& a_{0}(\lambda)=\lambda_{2}+\lambda_{3}+2 \lambda_{4}+2 \lambda_{5}+3 \lambda_{6}+3 \lambda_{7}+\cdots=\left|\lambda^{\prime}\right|+a_{1}\left(\lambda^{\prime}\right) \\
& a_{\ell}(\lambda)+c_{\ell}(\lambda)=a_{\ell-1}(\lambda)
\end{aligned}
$$

Lemma 2.4 Assume $n \geq 3$ and Theorem 2.1 is established for $\Lambda_{n-1}^{+}$, and take any $\lambda \in \Lambda_{n}^{+}$. Then the highest degree $\operatorname{hdeg}\left(p^{i} N_{i}\left(\lambda^{\prime}\right)\right)$ is strictly monotone increasing for $i \in\left[0, e v_{\lambda}\right]$, stable for $i \in\left[e v_{\lambda}, o d_{\lambda}-\lambda_{1}+\lambda_{2}\right]$ with value $a_{0}(\lambda)$, and strictly monotone decreasing for $i \in\left[\operatorname{od}_{\lambda}-\lambda_{1}+\lambda_{2},\left|\lambda^{\prime}\right|\right]$. 
Proof. We may use the result of Theorem 2.1 for $\lambda^{\prime} \in \Lambda_{n-1}^{+}$.

For $i \in\left[0, c_{-1}\left(\lambda^{\prime}\right)\right]$, the highest degree $\operatorname{hdeg}\left(p^{i} N_{i}\left(\lambda^{\prime}\right)\right)$ is strictly monotone increasing and takes the highest value at $c_{-1}\left(\lambda^{\prime}\right)=e v_{\lambda}$ with value

$$
c_{-1}\left(\lambda^{\prime}\right)+a_{0}\left(\lambda^{\prime}\right)=c_{1}(\lambda)+a_{1}(\lambda)=a_{0}(\lambda)
$$

by (2.23) and (2.25).

For $i \in J_{-1}\left(\lambda^{\prime}\right)=\left[c_{-1}\left(\lambda^{\prime}\right), c_{-2}\left(\lambda^{\prime}\right)\right]$, the highest degree $\operatorname{hdeg}\left(p^{i} N_{i}\left(\lambda^{\prime}\right)\right)$ is stable with value

$$
\operatorname{hdeg}\left(p^{i} N_{i}\left(\lambda^{\prime}\right)\right)=\left|\lambda^{\prime}\right|+a_{1}\left(\lambda^{\prime}\right)=a_{0}(\lambda),
$$

by (2.24), and

$$
c_{-2}\left(\lambda^{\prime}\right)=\left|\lambda^{\prime}\right|-c_{2}\left(\lambda^{\prime}\right)=\operatorname{od}_{\lambda}-\lambda_{1}+\lambda_{2} .
$$

For $i \in\left[c_{-2}\left(\lambda^{\prime}\right),\left|\lambda^{\prime}\right|\right]$, the highest degree hdeg $\left(p^{i} N_{i}\left(\lambda^{\prime}\right)\right)$ is strictly monotone decreasing and takes the highest value at $i=c_{-2}\left(\lambda^{\prime}\right)$ with value

$$
-c_{-2}\left(\lambda^{\prime}\right)+2\left|\lambda^{\prime}\right|+a_{2}\left(\lambda^{\prime}\right)=\left|\lambda^{\prime}\right|+c_{2}\left(\lambda^{\prime}\right)+a_{2}\left(\lambda^{\prime}\right)=a_{0}(\lambda)
$$

by (2.24) and (2.25).

Proof of Theorem 2.1. We assume that the statement holds for $n-1$, and take any $\lambda \in \Lambda_{n}^{+}$and fix it. We set

$$
(\text { pos })_{k}=\sum_{i=0}^{k} p^{i} N_{i}\left(\lambda^{\prime}\right), \quad(\text { neg })_{k}=\sum_{i=|\lambda|+1-k}^{\left|\lambda^{\prime}\right|} p^{i} N_{i}\left(\lambda^{\prime}\right)
$$

where $(n e g)_{k}$ appears only when $k>\lambda_{1}$. By Lemma 2.4, we see

$$
\begin{aligned}
\operatorname{hdeg}\left((\text { pos })_{k}\right)=a_{0}(\lambda) & \Longleftrightarrow k \geq e v_{\lambda}, \\
\operatorname{hdeg}\left((\text { neg })_{k}\right)=a_{0}(\lambda) & \Longleftrightarrow k>\lambda_{1} \text { and }|\lambda|-k+1 \leq o d_{\lambda}-\lambda_{1}+\lambda_{2} \\
& \Longleftrightarrow k \geq e v_{\lambda}+\lambda_{1}-\lambda_{2}+1 .
\end{aligned}
$$

Here we note that the condition (2.31) yields $k>\lambda_{1}$ and $k>e v_{\lambda}$.

Case $1\left[k \leq e v_{\lambda}\right]$

There exists an $\ell \geq 1$ such that $k \in J_{\ell-1}\left(\lambda^{\prime}\right)=J_{\ell}(\lambda)$ and

$$
\begin{aligned}
\operatorname{hterm}\left((\text { pos })_{k}\right) & =\operatorname{hterm}\left(p^{k} N_{k}\left(\lambda^{\prime}\right)\right)=C\left(n-1, \lambda^{\prime} ; k\right) p^{\ell k+a_{\ell-1}\left(\lambda^{\prime}\right)} \\
& =C\left(n-1, \lambda^{\prime} ; k\right) p^{\ell k+a_{\ell}(\lambda)} .
\end{aligned}
$$

If $k \leq \lambda_{1}$, (2.32) coincides with hterm $\left(N_{k}(\lambda)\right)$. Assume $k>\lambda_{1}$. Then, since $c_{\ell-1}\left(\lambda^{\prime}\right) \geq k$,

$|\lambda|-k+1 \geq|\lambda|-c_{\ell-1}\left(\lambda^{\prime}\right)+1=\left|\lambda^{\prime}\right|-\left(c_{(\ell-1)}\left(\lambda^{\prime}\right)-\lambda_{1}-1\right) \geq c_{-(\ell+1)}\left(\lambda^{\prime}\right)$, 
and

$$
\begin{aligned}
& \left.\operatorname{hdeg}\left((\text { neg })_{k}\right)\right)=\operatorname{hdeg}\left(p^{|\lambda|-k+1} N_{|\lambda|-k+1}\left(\lambda^{\prime}\right)\right) \\
& \quad \leq-\ell(|\lambda|-k+1)+(\ell+1)\left|\lambda^{\prime}\right|+a_{\ell+1}\left(\lambda^{\prime}\right)=-\ell \lambda_{1}+\left|\lambda^{\prime}\right|+\ell(k-1)+a_{\ell+2}(\lambda) .
\end{aligned}
$$

Hence, by (2.32) and (2.33), we have

$$
\begin{aligned}
\left.\left.\operatorname{hdeg}\left((\text { pos })_{k}\right)\right)-\operatorname{hdeg}\left((\text { neg })_{k}\right)\right) & \geq a_{\ell}(\lambda)-a_{\ell+2}(\lambda)-\left|\lambda^{\prime}\right|+\ell \lambda_{1}+\ell \\
& =-\left(\lambda_{2}+\cdots+\lambda_{\ell+1}\right)+\ell \lambda_{1}+\ell>0
\end{aligned}
$$

Hence, independently whether $k>\lambda_{1}$ or not, we have for $k \leq e v_{\lambda}$,

$$
\begin{aligned}
\operatorname{hterm}\left(N_{k}(\lambda)\right) & =\operatorname{hterm}\left(p^{k} N_{k}\left(\lambda^{\prime}\right)\right) \\
& =C\left(n-1, \lambda^{\prime} ; k\right) p^{\ell k+a_{\ell}(\lambda)} \quad\left(k \in J_{\ell}(\lambda), \ell \geq 1\right) .
\end{aligned}
$$

Further, if $k \in J_{\ell}(\lambda)$ with $\ell \geq 2$, then we see

$$
\begin{aligned}
C(n, \lambda ; k) & =C\left(n-1, \lambda^{\prime} ; k\right)=C\left(n-1-(\ell-1), \lambda^{\prime(\ell-1)} ; k\right) \\
& =C\left(n-\ell, \lambda^{(\ell)} ; k\right),
\end{aligned}
$$

and in particular,

$$
C\left(n, \lambda ; c_{\ell}(\lambda)\right)=C\left(n-1, \lambda^{\prime} ; c_{\ell-1}\left(\lambda^{\prime}\right)\right)=1, \quad \ell \geq 2
$$

Case $2\left[k \geq o d_{\lambda}\right]$

Since $N_{k}(\lambda)=N_{|\lambda|-k}(\lambda)$ and $|\lambda|-k \leq e v_{\lambda}$, the result follows from Case1. Thus, for some $\ell \geq 1, k \in J_{-\ell}(\lambda)$ and $|\lambda|-k \in J_{\ell}(\lambda)$, and

$$
\begin{aligned}
\operatorname{hterm}\left(N_{k}(\lambda)\right) & =C(n, \lambda ;|\lambda|-k) p^{\ell(|\lambda|-k)+a_{\ell}(\lambda)} \\
& =C\left(n-\ell, \lambda^{(\ell)} ;|\lambda|-k\right) p^{\ell(|\lambda|-k)+a_{\ell}(\lambda)} .
\end{aligned}
$$

Case $3\left[e v_{\lambda} \leq k \leq e v_{\lambda}+\lambda_{1}-\lambda_{2}\right]$

By (2.30) and (2.31), we have

$$
\begin{aligned}
& \operatorname{hdeg}\left(N_{k}(\lambda)\right)=\operatorname{hdeg}\left((\text { pos })_{k}\right)=a_{0}(\lambda), \\
& \text { htermcoeff }\left(N_{k}(\lambda)\right)=\sum_{i=e v_{\lambda}}^{\min \left\{k, o d_{\lambda}-\lambda_{1}+\lambda_{2}\right\}} C\left(n-1, \lambda^{\prime} ; i\right) .
\end{aligned}
$$

Case $4\left[e v_{\lambda}+\lambda_{1}-\lambda_{2}+1 \leq k \leq o d_{\lambda}\right]$

We see $\operatorname{hdeg}\left((\text { pos })_{k}\right)=\operatorname{hdeg}\left((\text { neg })_{k}\right)=a_{0}(\lambda)$ and $|\lambda|-k+1 \geq e v_{\lambda}+1$, and the difference of the highest coefficients becomes

$$
\begin{aligned}
& \text { htermcoeff }\left((\text { pos })_{k}\right)-\text { htermcoeff }\left((n e g)_{k}\right) \\
& =\sum_{i=e v_{\lambda}}^{\min \left\{k, o d_{\lambda}-\lambda_{1}+\lambda_{2}\right\}} C\left(n-1, \lambda^{\prime} ; i\right)-\sum_{i=|\lambda|-k+1}^{o d_{\lambda}-\lambda_{1}+\lambda_{2}} C\left(n-1, \lambda^{\prime} ; i\right) .
\end{aligned}
$$


Since $C\left(n-1, \lambda^{\prime} ; i\right)$ is symmetric for $i \in J_{-1}\left(\lambda^{\prime}\right)=\left[e v_{\lambda}, o d_{\lambda}-\lambda_{1}+\lambda_{2}\right]$ with respect to $\left(|\lambda|-\lambda_{1}+\lambda_{2}\right) / 2$ and

$$
\begin{aligned}
o d_{\lambda}-\lambda_{1}+\lambda_{2}-|\lambda|+k & =k-e v_{\lambda}-\lambda_{1}+\lambda_{2} \\
& <\min \left\{k, o d_{\lambda}-\lambda_{1}+\lambda_{2}\right\}-e v_{\lambda}+1
\end{aligned}
$$

we see (2.39) becomes

$$
\sum_{i=k-\lambda_{1}+\lambda_{2}}^{\min \left\{k, o d_{\lambda}-\lambda_{1}+\lambda_{2}\right\}} C\left(n-1, \lambda^{\prime} ; i\right)>0
$$

and $\operatorname{hdeg}\left(N_{k}(\lambda)\right)=a_{0}(\lambda)$ with coefficient (2.40).

By Case 3 and Case 4 , we obtain for $k \in J_{0}(\lambda)=\left[e v_{\lambda}, o d_{\lambda}\right]$,

$$
\begin{aligned}
& \operatorname{hterm}\left(N_{k}(\lambda)\right)=C(n, \lambda ; k) p^{a_{0}(\lambda)}, \\
& C(n, \lambda ; k)=\sum_{\max \left\{e v_{\lambda}, k-\lambda_{1}+\lambda_{2}\right\}}^{\min \left\{k, o d_{\lambda}-\lambda_{1}+\lambda_{2}\right\}} C\left(n-1, \lambda^{\prime} ; k\right) .
\end{aligned}
$$

By (2.41) and the induction hypothesis, the unimodality of $C(n, \lambda ; k)$ for $k \in J_{0}(\lambda)$ is clear, and one has for $c_{1}(\lambda)=e v_{\lambda}=c_{-1}\left(\lambda^{\prime}\right)$,

$$
C\left(n, \lambda ; c_{1}(\lambda)\right)=C\left(n-1, \lambda^{\prime} ; c_{1}(\lambda)\right)=C\left(n-1, \lambda^{\prime}, c_{-1}\left(\lambda^{\prime}\right)\right)=1,
$$

which completes the proof of Theorem 2.1 .

As a corollary of Theorem 2.1, we obtain a result for zeta functions, where we don't need the information of the leading coefficients, that of the highest degrees is enough.

Theorem 2.5 The zeta function $\zeta_{\lambda}(s)$ determines $\lambda \in \Lambda^{+}$.

Proof. The zeta function $\zeta_{\lambda}(s)$ determines $|\lambda|$ and $n$ for which $\lambda \in \Lambda_{n}^{+}$ (cf. (2.5)). Take $\lambda, \mu \in \Lambda_{n}^{+}$such that $\zeta_{\lambda}(s)=\zeta_{\mu}(s)$, then $\operatorname{hdeg}\left(N_{k}(\lambda)\right)=$ $\operatorname{hdeg}\left(N_{k}(\mu)\right)$ at any $k$. Since the statement for $n \leq 2$ is clear by (2.20) and (2.21), we consider the case $n \geq 3$. Assume $\lambda_{n}<\mu_{n}$. Taking $k=\lambda_{n}+1$, we have $(n-2)\left(\lambda_{n}+1\right)+\lambda_{n}=(n-1)\left(\lambda_{n}+1\right)$ by Theorem 2.1, which is a contradiction; thus $\lambda_{n}=\mu_{n}$. In the same way, we obtain $\lambda_{i}=\mu_{i}$ from $i=n-1$ to $i=1$ in order. Thus $\lambda=\mu$.

Since any finite abelian group is decomposed into the direct product of its Sylow $p$-subgroups, combining Theorem 2.5 and Proposition 1.2, we obtain the affirmative statement for Problem $[\mathrm{A}]$ :

Theorem 2.6 Within finite abelian groups, the zeta function $\zeta_{G}(s)$ determines the isomorphism class of $G$. 
Remark 2.7 The enumerating subgroups of finite abelian groups is a classical problem(cf. [Bu1], [Bu2]). The number $g_{\mu \nu}^{\lambda}(p)$ of subgroups $H$ of $G_{\lambda}$ such that $H \cong G_{\nu}$ and $G_{\lambda} / H \cong G_{\mu}$ was studied by G. Hall in 1950's, and it is known to have integral coefficients as a polynomial in $p$. These numbers $g_{\mu \nu}^{\lambda}(p)$ appear as coefficients of the product of Hall-Littlewood symmetric polynomials $P_{\lambda}(x ; t)$, which can be considered as a system of orthogonal polynomials associated with the root system of type $A_{n}$, and I. G. Macdonald have introduced and studied more general orthogonal polynomials associated with various root systems(cf. [Mac1], Mac2]). The unimodality of $N_{k}(\lambda)$ is proved by L. M. Butler by using a result on Hall-Littlewood polynomials([Bu1] $)$.

Remark 2.8 The zeta functions $\zeta_{\lambda}(s)$ has a close relation to Eisenstein series of $G L_{2 n}$ and local densities of square matrices, which we explain after [Sa]. Let $T \in M_{n}(\mathbb{Z})$ of rank $n$ and a prime $p$. Define

$$
b_{p}(T, s)=\int_{M_{n}\left(\mathbb{Q}_{p}\right)} \nu_{p}(X)^{-s} \mathbf{e}_{p}\left(2 \pi i \operatorname{tr}\left({ }^{t} T X\right)\right) d X,
$$

where $d X$ is the Haar measure on $M_{n}\left(\mathbb{Q}_{p}\right), \nu_{p}(X)$ is the product of the $p$ denominators of the elementary divisors of $X$ and $\mathbf{e}_{p}(x)=\exp \left(2 \pi i x^{\prime}\right), x^{\prime}$ being the fractional part. Then $b(T, s)=\prod_{p} b_{p}(T, s)$ appears as a main term in the Fourier expansion of $E_{n, n}(Y, s)$ for symmetric $Y \in M_{2 n}(\mathbb{Z})$, and $b(T, s)$ is an analogue of the Siegel singular series. The local density $\alpha_{p}\left(I_{m}, T\right)$ is defined by

$\alpha_{p}\left(I_{m}, T\right)=\lim _{\ell \rightarrow \infty} \frac{1}{p^{\ell\left(m^{2}-n^{2}\right)}} \sharp\left\{(X, Y) \in M_{m n}\left(\mathbb{Z} / p^{\ell} \mathbb{Z}\right)^{\oplus 2} \mid{ }^{t} X Y \equiv T \quad \bmod p^{\ell}\right\}$.

$b_{p}(T ; s)$ and $\alpha_{p}\left(I_{m}, T\right)$ are determined by the two-sided $G L_{n}\left(\mathbb{Z}_{p}\right)$-coset containing $T$, which is represented by some $D_{\lambda}=\operatorname{Diag}\left(p^{\lambda_{1}}, \ldots, p^{\lambda_{n}}\right)$ with $\lambda \in$ $\widetilde{\Lambda_{n}^{+}}=\left\{\lambda \in \mathbb{Z}^{n} \mid \lambda_{1} \geq \cdots \geq \lambda_{n} \geq 0\right\}$. Then it is known (cf. [Sa, Theorem 2], also $[\mathrm{BB}]$ )

$$
\begin{aligned}
& b_{p}\left(D_{\lambda}, s\right)=\zeta_{\lambda}(s-n) \cdot \prod_{i=0}^{n-1}\left(1-p^{-(s-i)}\right), \\
& b_{p}\left(D_{\lambda}, m\right)=\alpha_{p}\left(I_{m}, D_{\lambda}\right), \quad(m \geq n \geq 1) .
\end{aligned}
$$

Here $\lambda$ can be regarded in $\Lambda^{+}=\cup_{n} \Lambda_{n}^{+}$unless $\lambda=(0, \ldots, 0)$, which corresponds to the trivial group $\{e\}$ and $\zeta_{\{e\}}(s)=1$. By Theorem 2.5, we see $b_{p}\left(D_{\lambda}, s\right)$ is different for each $\lambda \in \widetilde{\Lambda_{n}^{+}}$. 


\section{$\S 3$}

We keep the notation in $\S 2$, especially $G_{\lambda}$ indicates the abelian $p$-group of order $p^{|\lambda|}$ defined in (2.1) for each $\lambda \in \Lambda^{+}$. We consider whether there is a group that is not isomorphic to $G_{\lambda}$ but has the same zeta function with $G_{\lambda}$ or not.

Proposition 3.1 (1) Let $\lambda \in \Lambda_{n}^{+}$and assume $n=1$ or $\lambda_{1}=1$. For a group $G$, the coincidence $\zeta_{G}(s)=\zeta_{\lambda}(s)$ implies the isomorphism $G \cong G_{\lambda}$.

(2) Let $\lambda \in \Lambda_{n}^{+}$with $n \geq 2$. Assume $\lambda_{1}>\lambda_{2}$ and $\lambda_{1} \geq 3$ if $p=2$. Then there exists a non-abelian group $\widetilde{G_{\lambda}}$ for which $\zeta_{\widetilde{G_{\lambda}}}(s)=\zeta_{\lambda}(s)$, e.g., set

$$
\widetilde{G_{\lambda}}=\left\langle a_{1}, \ldots, a_{n} \mid \begin{array}{l}
a_{i}^{p^{\lambda_{i}}}=1(1 \leq i \leq n), a_{n} a_{1} a_{n}^{-1}=a_{1}^{1+p^{\lambda_{1}-1}} \\
a_{i} a_{j}=a_{j} a_{i} \text { unless }\{i, j\}=\{1, n\}
\end{array}\right\rangle .
$$

Proof. (1) The result for the case $n=1$ follows from Proposition 1.1. Next, let $\lambda=\left(1^{n}\right), n \geq 2$ and assume the zeta function of a group $G$ coincides with $\zeta_{\lambda}(s)$. Since

$$
a_{p}(G)=N_{1}(\lambda)=\frac{p^{n}-1}{p-1},
$$

we see the exponent of $G$ is $p$. Denote by $Z(G)$ the center of $G$ and by $Z_{G}(a)$ the centralizer of $a(\in G)$ in $G$. Then any subgroup of $G$ of order $p^{2}$ is isomorphic to $C_{p} \times C_{p}$, and

$$
\begin{aligned}
a_{p^{2}}(G) & \leq \frac{(|Z(G)|-1)\left(p^{n}-p\right)}{\left(p^{2}-1\right)\left(p^{2}-p\right)}+\sum_{a \in G \backslash Z(G)} \frac{\left|Z_{G}(a)\right|-p}{\left(p^{2}-1\right)\left(p^{2}-p\right)} \\
& \leq \frac{\left(p^{r}-1\right)\left(p^{n}-p\right)+\left(p^{n}-p^{r}\right)\left(p^{n-1}-p\right)}{\left(p^{2}-1\right)\left(p^{2}-p\right)}
\end{aligned}
$$

where $p^{r}=|Z(G)|$. Hence

$$
\begin{aligned}
N_{2}(\lambda)-a_{p^{2}}(G) & \geq \frac{\left(p^{n}-1\right)\left(p^{n}-p\right)-\left\{\left(p^{r}-1\right)\left(p^{n}-p\right)+\left(p^{n}-p^{r}\right)\left(p^{n-1}-p\right)\right\}}{\left(p^{2}-1\right)\left(p^{2}-p\right)} \\
& =\frac{\left(p^{2 n}-p^{n+r}\right)\left(1-p^{-1}\right)}{\left(p^{2}-1\right)\left(p^{2}-p\right)} .
\end{aligned}
$$

Thus we see the identity $a_{p^{2}}(G)=N_{2}(\lambda)$ implies $r=n$, i.e., $G$ is abelian, hence $G \cong G_{\lambda}$ (the elementary abelian group of rank $n$ ).

(2) The strategy is similar to the case $G_{p}(m, n)$ in $\S 1$, indeed $G_{p}(m, n) \cong \widetilde{G_{\lambda}}$ with $\lambda=(m, n) \in \Lambda_{2}^{+}$. Take any $\lambda \in \Lambda_{n}^{+}$satisfying the condition of (2) and construct $\widetilde{G_{\lambda}}$. Any element $x \in \widetilde{G_{\lambda}}$ can be expressed uniquely as

$$
x=a_{1}^{e_{1}} \cdots a_{n}^{e_{n}}, \quad 0 \leq e_{i}<p^{\lambda_{i}}(1 \leq i \leq n),
$$

and

$$
\operatorname{ord}(x)=p^{\lambda_{1}} \quad \text { if and only if } p \nmid e_{1} .
$$


Further we see

$$
\begin{aligned}
& x^{r}=a_{1}^{e_{1}\left(r+\frac{r(r-1)}{2} e_{n} p^{\lambda_{1}-1}\right)} \prod_{i \geq 2} a_{i}^{r e_{i}}, \quad(r \geq 1), \\
& a_{n} x a_{n}^{-1}=a_{1}^{e_{1}\left(1+p^{\lambda_{1}-1}\right)} a_{2}^{e_{2}} \cdots a_{n}^{e_{n}}=x^{1+p^{\lambda_{1}-1}} .
\end{aligned}
$$

Thus, for any $x \in \widetilde{G_{\lambda}}$ of order $p^{\lambda_{1}},\langle x\rangle$ is normal in $\widetilde{G_{\lambda}}, \widetilde{G_{\lambda}} /\langle x\rangle \cong C_{p^{\lambda_{2}}} \times$ $\cdots \times C_{p^{\lambda_{n}}}$, and the set of subgroups of $\widetilde{G_{\lambda}}$ containing $\langle x\rangle$ is one to one corresponding to the set of subgroups of $C_{p^{\lambda_{2}}} \times \cdots \times C_{p^{\lambda_{n}}}$. On the other hand, any subgroup of $\widetilde{G_{\lambda}}$ without an element of order $p^{\lambda_{1}}$ is contained in $\left\langle a_{1}{ }^{p}, a_{2}, \cdots, a_{n}\right\rangle \cong C_{p^{\lambda_{1}-1}} \times C_{p^{\lambda_{2}}} \times \cdots \times C_{p^{\lambda_{n}}}$. The above situation is similar to the case of $G_{\lambda}$, and we see there is a natural bijection between the set of subgroups of $\widetilde{G_{\lambda}}$ and that of $G_{\lambda}$, and their zeta functions coincide.

Remark 3.2 The zeta function of the above group $\widetilde{G_{\lambda}}$ satisfies the symmetry (0.3), by virtue of its coincidence with that of $G_{\lambda}$. Hence, one has many examples of non-abelian groups within nilpotent groups having zeta functions with symmetry. By neumerical data, we know some examples of non-abelian groups whose zeta functions do not coincide with that of any abelian group but satisfies the symmetry, e.g., the group of GAP ID $\left(2^{4}, 10\right)$ (the smallest order) or GAP ID $\left(3^{4}, 14\right)$ (the smallest odd order).

Let $\lambda \in \Lambda_{n}^{+}$with $n \geq 2$. Assume $\lambda \neq(1, \ldots, 1)$ and also $\lambda \neq(2,1, \ldots, 1)$ if $p=2$. Then, we expect there exists a non-abelian $p$-group for which $\zeta_{G}(s)=\zeta_{\lambda}(s)$.

Acknowledgement: The author expresses her thanks to A. Hoshi who checked the groups of order 27 by using GAP. She is thankful also to the members of WINJ, since WINJ7 was a good opportunity to think about this theme.

\section{References}

[BB] J. Beineke and D. Bump: A certain formula for divisor functions associated to lattices, Forum Math. 18 (2006), 869 - 906.

[Bu1] L. M. Butler: A unimodality result in the enumeration of subgroups of a finite groups, Proc. Amer. Math. Soc. 101(1987), $771-775$.

[Bu2] L. M. Butler: Subgroup lattices and symmetric functions, Memoires Amer. Math. Soc. 539(1994). 
[GSS] F. J. Grunewald, D. Segal and G. C. Smith, Subgroups of finite index in nilpotent groups, Invent. math.93(1988), $185-223$.

[Mac1] I. G. Macdonald: Symmetric functions and Hall polynomials, Oxford Univ. Press (1979).

[Mac2] I. G. Macdonald: Orthogonal polynomials associated with root systems, Séminaire Lotharingien de Combinatoire 45(2000), Article B45a.

[Sau] M. P. F. du Sautoy: Finitely generated groups, p-adic analytic groups and Poincaré series, Ann. Math. 137(1993), 639-670.

[SG] M. P. F. du Sautoy and F. J. Grunewald: Analytic properties of zeta functions and subgroup growth, Ann. Math. 152(2000), 793 - 833.

[Sa] F. Sato: Fourier coefficients of Eisenstein series of $G L_{n}$, local densities of square matrices and subgroups of finite abelian groups, Comment. Math. Univ. St. Pauli 55(2006), 77 - 95.

[St] T. Stehling: On computing the number of subgroups of a finite abelian group, Combinatorica 12(1992), 475 - 479. 